\title{
The Power Counting Theorem for Feynman Integrals with Massless Propagators
}

\author{
J. H. Lowenstein \\ Physics Department, New York University, New York, N. Y., USA \\ W. Zimmermann \\ Max-Planck-Institut für Physik und Astrophysik, München, Federal Republic of Germany
}

Received March 27, 1975

\begin{abstract}
Dyson's power counting theorem is extended to the case where some of the mass parameters vanish. Weinberg's ultraviolet convergence conditions are supplemented by infrared convergence conditions which combined are sufficient for the convergence of Feynman integrals.
\end{abstract}

\section{Introduction}

In the theory of renormalization Dyson's power counting theorem plays a decisive part [1-3]. The contribution of a proper Feynman diagram to a Green's function has the form

where

$$
\begin{aligned}
& J=\frac{\int d k R(k, p)}{\prod_{j=1}^{n}\left(l_{j}^{2}-m_{j}^{2}+i \varepsilon\left(\vec{l}_{j}^{2}+m_{j}^{2}\right)\right)^{n_{j}}}
\end{aligned}
$$

$$
\begin{array}{cc}
k=\left(k_{1} \ldots k_{m}\right), & p=\left(p_{1} \ldots p_{N}\right), \\
k_{j}=\left(k_{j 0} k_{j 1} k_{j 2} k_{j 3}\right), & p_{j}=\left(p_{j 0} p_{j 1} p_{j 2} p_{j 3}\right), \\
d k=d k_{1} \ldots d k_{m}, & d k_{j} \ldots d k_{j 0} d k_{j 1} d k_{j 2} d k_{j 3}, \\
m_{j} \geqq 0, \quad n_{j}>0 . &
\end{array}
$$

$k_{j}$ and $p_{j}$ are Minkowski vectors with the metric $(+1,-1,-1,-1)$. The vectors $l_{j}$ are linear combinations

$$
l_{j}=K_{j}(k)+P_{j}(p)
$$

of the vectors $k_{1}, \ldots, k_{m}$ and $p_{1}, \ldots, p_{N}$ with $K_{j} \neq 0 . P$ is a polynomial in the components of $k$ and $p$. The denominator of $R$ is the common denominator of the unrenormalized integrand and the subtraction terms.

If all masses are non-zero Weinberg's version of the power counting theorem can be used to prove that the integral (1.1) is absolutely convergent provided the renormalized integrand $R$ has been constructed according to Bogoliubov's subtraction rules $[3,4]$. It can further be shown that the limit $\varepsilon \rightarrow+0$ exists as a covariant tempered distribution.

So far the power counting theorem has only been stated for non-vanishing masses. In the present paper Weinberg's ultraviolet convergence conditions are 
supplemented by infrared convergence conditions which will be shown to be sufficient for the convergence of integrals (1.1). The limit $\varepsilon \rightarrow+0$ [5] as well as the application to field theoretic models [6-9] are discussed in separate papers.

Our results are consistent with recent work by Bergère and Lam, as well as by Trute and Pohlmeyer, on the asymptotic behavior of parametrized Feynman integrals for small mass values [10-12].

Some general definitions are given in Section 2. Section 3 contains the statement and proof of the power counting theorem. The concept of reduced integrals, which is useful for some application of the theorem, is introduced in Section 4.

\section{General Definitions}

We consider integrals of the form (1.1). $L$ denotes the space of the linear forms

$$
l=\sum_{j=1}^{n} a_{j} k_{j}+\sum_{j=1}^{N} b_{j} p_{j}
$$

which will be interpreted as inhomogeneous linear forms in the integration variables $k_{1}, \ldots, k_{m}$. Elements of $L$ are called linerarly (in)dependent if their homogeneous parts (in $k$ ) are linearly (in)dependent. A set of elements in $L$ is called a basis of $L$ if their homogeneous parts form a basis for the space of the homogeneous forms in $k$.

We observe that always for an absolutely convergent integral (1.1) a basis

$$
l_{j_{1}} \ldots, l_{j_{m}}
$$

exists consisting of linear forms which occur in the denominators of (1.1). Otherwise there would be at most $m^{\prime}<m$ linearly independent forms

$$
l_{j_{1}}, \ldots, l_{j_{m^{\prime}}}
$$

with the remaining $l_{j}$ being linear combinations of vectors (2.3) and $p_{j}$. Extending (2.3) to a basis

$$
l_{j_{1}}, \ldots, l_{j_{m^{\prime}}}, w_{1}, \ldots, w_{c} \quad m^{\prime}+c=m
$$

of $L$ with Jacobian one (relative to $k_{1}, \ldots, k_{m}$ ) we find

$$
J=\int d l_{j_{1}} \ldots d l_{j_{m^{\prime}}} d w_{1} \ldots d w_{c} R
$$

with the divergent subintegral

$$
\int d w_{1} \ldots d w_{c} R=\frac{1}{\prod\left(l_{j}^{2}-m_{j}^{2}+\ldots\right)^{n_{j}}} \int d w_{1} \ldots d w_{c} P .
$$

Therefore, a basis (2.2) of $L$ must always exist if the integral (1.1) is to be absolutely convergent.

For the formulation of the power counting theorem we will need certain subintegrals which we set up as follows. Let

$$
u_{1}=l_{i_{1}}, \ldots, u_{a}=l_{i_{a}}, v_{1}=l_{j_{1}}, \ldots, v_{b}=l_{j_{b}}
$$

be a basis of $L$ with Jacobian one (relative to $k_{1}, \ldots, k_{m}$ ). Using (2.4) as new integration variables for (1.1) we obtain

$$
\begin{array}{rlrl}
J & =\int d u d v R, & \\
u & =\left(u_{1} \ldots u_{a}\right), & v & =\left(v_{1} \ldots v_{b}\right), \\
d u & =d u_{1} \ldots d u_{a}, & d v & =d v_{1} \ldots d v_{b}
\end{array}
$$


where $P$ and $l_{j}$ are expressed in terms of $u, v$ and $p$ through

$$
k=k(u, v, p) .
$$

We consider a hyperplane $H$ defined by the condition that the linear forms

$$
v_{1}=l_{j_{1}}, \ldots, v_{b}=l_{j_{b}}
$$

have constant values. The subintegral of (1.1) along $H$ is then given by

$$
J(H)=\int d u R .
$$

We distinguish two different definitions for the dimension of a subintegral (2.6).

The upper dimension $\overline{\mathrm{dim}}$ refers to the behavior for large values of the integration variables. The lower dimension dim refers to the behavior for small values of the integration variables. We define

$$
\begin{aligned}
& \overline{\operatorname{dim}} J(H)=\overline{\operatorname{deg}}_{u} R+4 a, \\
& \underline{\operatorname{dim}} J(H)=\underline{\operatorname{deg}}_{u} R+4 a .
\end{aligned}
$$

The upper degree $\overline{\operatorname{deg}}_{u}$ (or lower degree $\operatorname{deg}_{u}$ ) denotes the leading power of $\varrho$ in the limit $\varrho \rightarrow \infty$ (or $\varrho \rightarrow 0$ ) if $u_{j}=\varrho \hat{u}_{j}$ is substituted into $R$. More precisely,

$$
\bar{v}=\overline{\operatorname{deg}}_{u} R, \quad \underline{y}=\underline{\operatorname{deg}}_{u} R,
$$

if

$$
\lim _{\varrho \rightarrow \infty} \frac{R}{\varrho^{\bar{v}}} \neq 0, \infty, \quad \lim _{\varrho \rightarrow 0} \frac{R}{\varrho^{\underline{v}}} \neq 0, \infty
$$

for almost all values of $u_{1}, \ldots, u_{a}$ and the remaining parameters $v_{1}, \ldots, v_{b}, p_{1}, \ldots, p_{N}$.

We quote some rules for the upper and lower degree. Let $N, D, F, F_{1}, \ldots, F_{r}$ be complex-valued functions of real four-vectors $u_{1}, \ldots, u_{a}, v_{1}, \ldots, v_{b}, p_{1}, \ldots, p_{N}$ to which the definitions $\overline{\operatorname{deg}}_{u}$ and $\operatorname{deg}_{u}$ may be applied. Then the following rules hold

$$
\begin{aligned}
& \overline{\operatorname{deg}}_{u} F^{n}=n \overline{\operatorname{deg}}_{u} F, \\
& {\overline{\operatorname{deg}_{u}}}^{n}=n \overline{\operatorname{deg}}_{u} F, \\
& \overline{\operatorname{deg}}_{u} \frac{N}{D}=\overline{\operatorname{deg}}_{u} N-\overline{\operatorname{deg}}_{u} D, \\
& {\underline{\operatorname{deg}_{u}}}_{D} \frac{N}{D}=\underline{\operatorname{deg}}_{u} N-\underline{\operatorname{deg}}_{u} D, \\
& \overline{\operatorname{deg}}_{u} \prod_{j=1}^{r} F_{j}=\sum_{j=1}^{r} \overline{\operatorname{deg}_{u} F_{j},} \\
& \underline{\operatorname{deg}}_{u} \prod_{j=1}^{r} F_{j}=\sum_{j=1}^{r} \underline{\operatorname{deg}_{u} F_{j},} \\
& \overline{\operatorname{deg}}_{u} \sum_{j=1}^{r} F_{j} \leqq \max _{j}\left\{\overline{\operatorname{deg}}_{u} F_{j}\right\}, \\
& \underline{\operatorname{deg}}_{u} \sum_{j=1}^{r} F_{j} \geqq \min \left\{\underline{\operatorname{deg}}_{j} F_{j}\right\} .
\end{aligned}
$$


Let $F$ be a polynomial of $u=\left(u_{1}, \ldots, u_{a}\right), v=\left(v_{1}, \ldots, v_{b}\right)$ and $p=\left(p_{1}, \ldots, p_{N}\right)$ with vectors $u_{i}, v_{j}, p_{r}$. Then we may write

$$
F=\sum_{\alpha} Q_{\alpha} M_{\alpha}, \quad Q_{\alpha} \neq 0
$$

where $M_{\alpha}$ are independent monomials in $u$ and $Q_{\alpha}$ are polynomials in $v, p$ which are not identically zero. The upper and lower degrees of $F$ are given by

$$
\begin{aligned}
& \overline{\operatorname{deg}}_{u} F=\max _{\alpha}\left\{\operatorname{deg} M_{\alpha}\right\}, \\
& \overline{\operatorname{deg}}_{u} F=\min _{\alpha}\left\{\operatorname{deg} M_{\alpha}\right\} .
\end{aligned}
$$

\section{Convergence Theorem}

In this section the power counting theorem will be formulated for integrals $J$ of type (1.1) assuming that a basis (2.2) of $L$ can be formed. Weinberg's hypothesis of the power counting theorem may be stated as follows:

Ultraviolet Convergence Condition. The inequality

$$
\overline{\operatorname{dim}} J(H)=\overline{\operatorname{deg}}_{u} R+4 a<0
$$

holds for any basis (2.4) and for any hyperplane $H$ defined by constant values of $v_{1}, \ldots, v_{b}$.

In particular, the upper dimension of the full integral $J$ should be negative. Weinberg's condition (3.1) is sufficient for the absolute convergence of $J$ provided all masses are different from zero

$$
m_{j}>0, \quad j=1, \ldots, n .
$$

In the general case we propose in addition the following

Infrared Convergence Condition. The inequality

$$
\underline{\operatorname{dim}} J(H)=\underline{\operatorname{deg}}_{u} R+4 a>0
$$

holds for any basis (2.4) satisfying

$$
m_{i_{1}}=\ldots=m_{i_{a}}=0
$$

and for any hyperplane $H$ defined by constant values of $v_{1}, \ldots, v_{b}$.

The ultraviolet and infrared convergence conditions combined form the hypothesis of the

Power Counting Theorem. Let $J$ be an integral of the form (1.1) for which a basis (2.2) of $L$ can be formed. $J$ is absolutely convergent if the ultraviolet convergence condition (3.1) and the infrared convergence condition (3.2-3) hold.

Due to the inequality

$$
\frac{l_{0}^{2}+\vec{l}^{2}+m^{2}}{\left|l_{0}^{2}-\vec{l}^{2}-m^{2}+i \varepsilon\left(\vec{l}^{2}+m^{2}\right)\right|} \leqq\left(1+\frac{4}{\varepsilon^{2}}\right)^{\frac{1}{2}}
$$


the absolute convergence of (1.1) is implied by the absolute convergence of the corresponding Euclidean integral

$$
\begin{aligned}
& J=\int d k R(k, p) \\
& R=\frac{P}{\prod_{j=1}^{n}\left(l_{j}^{2}+m_{j}^{2}\right)^{n_{j}}}
\end{aligned}
$$

where now

$$
l_{j}^{2}=l_{j 0}^{2}+\vec{l}_{j}^{2} .
$$

Therefore, we may restrict ourselves to proving the absolute convergence of (3.4) under the conditions (3.1-3).

We begin proving a lemma on the infrared convergence of certain integrals which are homogeneous in the integration variables.

Lemma. Consider integrals of the form

$$
F=\int_{u_{i}^{2} \leqq 1} d u_{1} \ldots d u_{a} \frac{M}{\prod_{j}\left(U_{j}^{2}\right)^{n_{j}}}
$$

where the $U_{j}$ are linear combinations of the Euclidean four-vectors $u_{1}, \ldots, u_{a}$ and $M$ is a monomial in the components of $u_{1}, \ldots, u_{a} . M$ may be factorized as

$$
M=\prod_{i=1}^{a} M_{i}
$$

where $M_{i}$ is a monomial of $u_{i}$. For any subset

$$
u_{i_{1}}, \ldots, u_{i_{c}}
$$

of the integration variables we form the integral

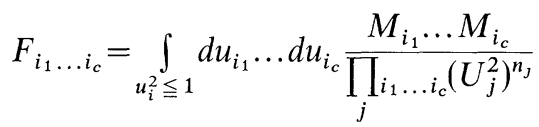

where the product $\prod_{j} i_{1} \ldots i_{c}$ extends over all $U_{j}$ which are linear combinations of vectors (3.7) only. The integrals (3.8) are called sections of (3.5).

The statement is that the integral (3.5) is absolutely convergent if the dimension $d_{i_{1} \ldots i_{c}}$ of each section (3.8) is positive:

$$
\operatorname{dim} F_{i_{1} \ldots i_{c}}=d_{i_{1} \ldots i_{c}}>0 .
$$

This condition includes the dimension of the full integral which we denote by $d$,

$$
d=\operatorname{dim} F=d_{1 \ldots a}>0 .
$$

Proof. We decompose the integral (3.5) into

$$
\begin{aligned}
F & =\sum_{P} F_{P} \\
F_{P} & =\int_{u_{i_{1}}^{2} \leqq \ldots \leqq u_{i_{a}}^{2} \leqq 1} d u_{1} \ldots d u_{a} \frac{M}{\prod_{j}\left(U_{j}^{2}\right)^{n_{j}}}
\end{aligned}
$$


with the sum extending over all permutations

$$
P=\left(\begin{array}{c}
1 \ldots a \\
i_{1} \ldots i_{a}
\end{array}\right) \text {. }
$$

We will check the convergence of each term $F_{P}$. In order to simplify the notation we rename the integration variables and monomials by

$$
\begin{gathered}
w_{1}=u_{i_{1}}, \ldots, w_{a}=u_{i_{a}}, \\
N_{1}=M_{i_{1}}, \ldots, N_{a}=M_{i_{a}} .
\end{gathered}
$$

Moreover, we denote the momenta $U_{j}$ and exponents $n_{j}$ of the denominators by

$$
\begin{array}{r}
W_{11}, \ldots, W_{21}, \ldots, \ldots, W_{a 1}, \ldots \\
n_{11}, \ldots, n_{21}, \ldots, \ldots, n_{a 1}, \ldots
\end{array}
$$

such that each $W_{i j}$ is a linear combination of $w_{1}, \ldots, w_{i}$ with non-vanishing coefficient of $w_{i^{\prime}}$

$$
W_{i j}=\sum_{i^{\prime}=1}^{i} c_{i j i^{\prime}} w_{i^{\prime}}, \quad c_{i j i} \neq 0 .
$$

In this notation $F_{P}$ may be written in the form

$$
F_{P}=\int_{w_{1}^{2} \leqq 1} d w_{1} \frac{N_{1}}{\prod_{j}\left(W_{1 j}^{2}\right)^{n_{1} j}} \int_{w_{1}^{2} \leqq w_{2}^{2} \leqq 1} d w_{2} \frac{N_{2}}{\prod_{j}\left(W_{2 j}^{2}\right)^{n_{2}}} \cdots \int_{w_{a-1}^{2} \leqq w_{a}^{2} \leqq 1} d w_{a} \frac{N_{a}}{\prod_{j}\left(W_{a j}^{2}\right)^{n_{a j}}} .
$$

According to the hypothesis of the Lemma the dimension $d_{c}$ of each section

$$
F_{c}=F_{i_{1} \ldots i_{c}}=\int_{w_{1}^{2} \leqq 1} d w_{1} \frac{N_{1}}{\prod_{j}\left(W_{1 j}^{2}\right)^{n_{1}}} \ldots \int_{w_{c}^{2} \leqq 1} d w_{c} \frac{N_{c}}{\prod_{j}\left(W_{c j}^{2}\right)^{n_{c j}}}
$$

is positive,

$$
\operatorname{dim} F_{c}=d_{c}>0 .
$$

$d_{c}$ satisfies the recursion formula

$$
d_{c}=4+\operatorname{deg} N_{c}-\operatorname{deg} \prod_{j}\left(W_{c j}^{2}\right)^{n_{c j}}+d_{c-1} .
$$

We choose a number $\delta$ with

$$
d_{c}>\delta>0 \text { for } c=1, \ldots, a
$$

and form the integral

$$
G=\int_{w_{1}^{2} \leqq 1} d w_{1} \frac{\left|N_{1}\right|}{\prod_{j}\left(W_{1 j}^{2}\right)^{n_{1 j}}} \int_{w_{1}^{2} \leqq w_{2}^{2} \leqq 1} d w_{2} \frac{\left|N_{2}\right|}{\prod_{j}\left(W_{2 j}^{2}\right)^{n_{2 j}}} \ldots \int_{w_{a-1}^{2} \leqq w_{a}^{2} \leqq 1} d w_{a} \frac{\left|N_{a}\right|}{\left|w_{a}\right|^{d-\delta} \prod_{j}\left(W_{a j}^{2}\right)^{n_{a j}}}
$$

Since $\left|w_{a}\right| \leqq 1$ and $d-\delta=d_{a}-\delta>0$ the integral $F_{P}$ is majorized by $G$,

$$
\left|F_{P}\right| \leqq G \text {. }
$$


We will prove the convergence of $G$ by recursively estimating the integrals

$$
G_{c}=\int_{w_{c}^{2} \leqq w_{c+1}^{2} \leqq 1} d w_{c+1} \frac{\left|N_{c}\right|}{\prod_{j}\left(W_{c+1, j}^{2}\right)^{n_{c+1, j}}} \ldots \int_{w_{a-1}^{2} \leqq w_{a}^{2} \leqq 1} d w_{a} \frac{\left|N_{a}\right|}{\left|w_{a}\right|^{d-\delta} \prod_{j}\left(W_{a j}^{2}\right)^{n_{a j}}} .
$$

The dimension of

$$
G_{a-1}=\int_{w_{a-1}^{2} \leqq w_{a}^{2} \leqq 1} d w_{a} \frac{\left|N_{a}\right|}{\left|w_{a}\right|^{d-\delta} \prod_{j}\left(W_{a j}^{2}\right)^{n_{a j}}}
$$

is [see Eq. (3.13)]

$$
\operatorname{dim} G_{a-1}=4+\operatorname{deg} N_{a}-\operatorname{deg} \prod_{j}\left(W_{a j}^{2}\right)^{n_{a j}}+\delta-d .
$$

Now, by a change of integration variable,

$$
G_{a-1}=\frac{1}{\left|u_{a-1}\right|^{\delta_{a-1}-d}} \int_{1 \leqq w_{a}^{2} \leqq \frac{1}{w_{a}^{2}-1}} d w_{a} \frac{\left|N_{a}\right|}{\left|w_{a}\right|^{d-\delta} \prod_{j} W_{a j}^{2}} .
$$

In the last line the limit $1 /\left|u_{a-1}^{2}\right| \rightarrow \infty$ could be performed since the dimension of the integral is negative. Hence

$$
G_{a-1} \leqq \frac{\gamma_{a-1}}{\left|u_{a-1}\right|^{d_{a-1}-\delta}}
$$

where $\gamma_{a-1}$ is a constant.

Repeating this argument recursively we obtain

$$
G_{c} \leqq \frac{\gamma_{c}}{\left|w_{c}\right|^{d_{c}-\delta}}
$$

by Eq. (3.13-14). Finally

$$
G \leqq \gamma \int_{w_{1}^{2} \leqq 1} d w_{1} \frac{\left|N_{1}\right|}{\left|w_{1}\right|^{d_{1}-\delta} \prod_{j}\left(W_{1 j}^{2}\right)^{n_{1 j}}} .
$$

The integral on the right hand side exists since its dimension

$$
\operatorname{dim} F_{1}-d_{1}+\delta=\delta>0
$$

is positive. By (3.16) each term of the decomposition (3.10) is absolutely convergent which implies the absolute convergence of (3.5). This completes the proof of the lemma.

We now turn to the

Proof of the Power Counting Theorem. Let $S_{0}$ be the set of all momenta $l_{j}$ with $m_{j}=0$. Let $S$ be any subset

$$
S \subseteq S_{0}
$$

$T$ denotes the complementary set

$$
T=S_{0} \backslash S \text {. }
$$


We require that with a momentum $l_{j}$ the set $S$ should contain any $l_{i}$ which satisfies

$$
l_{i}^{2} \equiv l_{j}^{2}, \quad m_{i}=0 .
$$

We decompose the integral (3.4) into

$$
J=\sum_{S} A_{S}
$$

where

$$
A_{S}=\int_{\substack{l^{2} \leqq \\ l_{l}^{2} \geqq r^{2} \text { in } T}} d k \frac{P}{\prod_{j}\left(l_{j}^{2}+m_{j}^{2}\right)^{n_{j}}} .
$$

For studying $A_{S}$ we select momentum vectors

$$
u_{1}=l_{i_{1}}, \ldots, u_{a}=l_{i_{a}}
$$

in $S$ which form a basis of $S$. Then $l_{j} \in S$ is a linear combination of $u_{1}, \ldots, p_{1}, \ldots$,

$$
\begin{aligned}
l_{i} & =U_{i}+Q_{i} \\
U_{i} & =\sum_{j=1}^{a} c_{i j} u_{j}, \quad Q_{i}=\sum_{j=1}^{N} d_{i j} p_{j} .
\end{aligned}
$$

We say that $S$ or the integral $A_{S}$ has zero external momenta if $Q_{i}=0$ for all $l_{i} \in S$.

For $r$ small enough the term $A_{S}$ vanishes unless all external momenta vanish. For the proof we observe that $u_{\alpha}^{2} \leqq r^{2}$ since the $u_{\alpha}$ occur among the $l_{i} \in S$. The $U_{j}$ are of the form

$$
U_{j}=\sum_{\alpha=1}^{a} \eta_{\alpha} u_{\alpha}
$$

where $\left|\eta_{\alpha}\right| \leqq \eta$ with $\eta$ being characteristic number of the integral. Now

$$
\left|Q_{j}\right| \leqq\left|l_{j}\right|+\left|U_{j}\right| \leqq(1+\eta) r
$$

implies

$$
r \geqq \frac{\left|Q_{j}\right|}{1+\eta} \text { for any } \quad Q_{j},
$$

if the domain of integration is not empty. If at least one $Q_{j} \neq 0$ we may choose $r$ such that

$$
0<r<\frac{\left|Q_{j}\right|}{1+\eta} .
$$

But then the domain of integration is empty and $A_{S}=0$. Hence for $r$ small enough we find

$$
J=\sum_{S} A_{S}
$$

where $S$ is restricted to those subsets for which $Q_{j}=0$ for any $l_{j} \in S$.

In each integral $A_{S}$ we introduce new variables of integration as follows. By adding suitable vectors

$$
v_{1}=l_{j_{1}}, \ldots, v_{b}=l_{j_{b}}, \quad a+b=m,
$$


we extend (3.20) to a basis

$$
u_{1}, \ldots, u_{a}, v_{1}, \ldots, v_{b}
$$

of $L$ with Jacobian one (relative to $k_{1}, \ldots, k_{m}$ ). Then each $l_{j} \in S$ is a linear combination of $u_{1}, \ldots, u_{a}$. The remaining $l_{j}$ are linear combinations of $u_{1}, \ldots, u_{a}, v_{1}, \ldots, v_{b}$ $p_{1}, \ldots, p_{N}$. We next write the numerator $P$ as a polynomial in $u$

$$
\begin{aligned}
& P=\sum C_{S \alpha} M_{\alpha}, \\
& M_{\alpha}=\prod_{i=1}^{a} M_{i \alpha_{\imath}}, \quad \alpha=\left(\alpha_{1}, \ldots, \alpha_{a}\right), \\
& M_{i \alpha_{i}}=u_{i 0}^{\alpha_{20}} u_{i 1}^{\alpha_{21}} u_{i 2}^{\alpha_{i 2}} u_{i 3}^{\alpha_{23}}, \quad \alpha_{i}=\left(\alpha_{i 0}, \ldots, \alpha_{i 3}\right) \text {. }
\end{aligned}
$$

with the coefficients being polynomials in $v_{1}, \ldots, v_{b}, p_{1}, \ldots, p_{N}$. Then

$$
\begin{aligned}
A_{S} & =\sum_{\alpha} A_{S \alpha}, \\
A_{S \alpha} & =\int_{l_{j}^{2} \leqq r^{2} \text { in } S} d u \frac{M_{\alpha}}{\prod_{S}\left(l_{j}^{2}\right)^{n_{j}}} \int d v \frac{C_{S \alpha}}{\prod_{T}\left(l_{j}^{2}\right)^{n_{J}} \prod_{U}\left(l_{j}^{2}+m_{j}^{2}\right)^{n_{J}}}, \\
U & =\left(l_{1}, \ldots, l_{n}\right) \backslash S_{0} .
\end{aligned}
$$

We now estimate the $v$-integrals

$$
\int_{l_{\imath}^{2} \geqq r^{2} \text { in } T} d v \frac{C_{S \alpha}}{\prod_{T}\left(l_{j}^{2}\right)^{n_{j}} \prod_{U}\left(l_{j}^{2}+m_{j}^{2}\right)^{n_{j}}} .
$$

To this end we consider the integral

$$
\int d v \frac{P}{\prod_{T}\left(l_{j}^{2}+M^{2}\right)^{n_{j}} \prod_{U}\left(l_{j}^{2}+m_{j}^{2}\right)^{n_{J}}}
$$

with $M>0$. In (3.28) all masses are different from zero. Because of the ultraviolet convergence conditions the integral (3.28) is absolutely convergent. Each $l_{j}$ in (3.28) is of the form

$$
l_{j}=V_{j}(v)+R_{j}(u, p) .
$$

Using

$$
\frac{l_{j}^{2}+m^{2}}{V_{j}^{2}+m^{2}} \leqq 1+\frac{\left|l_{j}-V_{j}\right|}{m}+\frac{\left|l_{j}-V_{j}\right|^{2}}{m^{2}}
$$

we find

$$
\begin{aligned}
& \int d v \frac{|P|}{\prod_{T}\left(V_{j}^{2}+M^{2}\right)^{n_{j}} \prod_{U}\left(V_{j}^{2}+M^{2}\right)^{n_{j}}} \\
& \quad \leqq c \int d v \frac{|P|}{\prod_{T}\left(l_{j}^{2}+M^{2}\right)^{n_{j}} \prod_{U}\left(l_{j}^{2}+M^{2}\right)^{n_{j}}} .
\end{aligned}
$$

Hence

$$
\int d v \frac{P}{\prod_{T}\left(V_{j}^{2}+M^{2}\right)^{n_{j}} \prod_{U}\left(V_{j}^{2}+m_{j}^{2}\right)^{n_{j}}}
$$


is absolutely convergent. The denominator does not depend on $u$ while the numerator is a polynomial in $u$. Applying Lemma 3 of Ref. [13] we find that

$$
\int d v \frac{C_{\alpha}(v, p)}{\prod_{T}\left(V_{j}^{2}+M^{2}\right)^{n_{j}} \prod_{U}\left(V_{j}^{2}+m_{j}^{2}\right)^{n_{j}}}
$$

is absolutely convergent. With this we can estimate (3.27):

$$
\begin{aligned}
& \int_{l_{j}^{2} \geq r^{2}} d v \frac{\left|C_{S \alpha}\right|}{\prod_{T}\left(l_{j}^{2}\right)^{n_{j}} \prod_{U}\left(l_{j}^{2}+m_{j}^{2}\right)^{n_{j}}} \\
& \leqq\left(1+\frac{M^{2}}{r^{2}}\right)^{\tau} \int d v \frac{\left|C_{\alpha}\right|}{\prod_{T}\left(l_{j}^{2}+M^{2}\right)^{n_{j}} \prod_{U}\left(l_{j}^{2}+M^{2}\right)^{n_{j}}} \\
& \quad\left(\text { using } \frac{l_{j}^{2}+M^{2}}{l_{j}^{2}} \leqq 1+\frac{M^{2}}{r^{2}}\right) \\
& \leqq d \int d v \frac{\left|C_{\alpha}\right|}{\prod_{T}\left(V_{j}^{2}+M^{2}\right)^{n_{j}} \prod_{U}\left(V_{j}^{2}+m_{j}^{2}\right)^{n_{j}}}
\end{aligned}
$$

using

$$
\frac{V_{j}^{2}+M_{j}^{2}}{l_{j}^{2}+M_{j}^{2}} \leqq 1+\frac{\left|l_{j}-V_{j}\right|}{M_{j}}+\frac{\left|l_{j}-V_{j}\right|^{2}}{M_{j}^{2}}
$$

The integral of the last line only depends on $p$ and the masses. Hence

$$
|J| \leqq \sum_{S \alpha} D_{S \alpha} \int_{l_{j}^{2} \leqq r^{2}} d u \frac{\left|M_{\alpha}\right|}{\prod_{s}\left(l_{j}^{2}\right)^{n(j)}} .
$$

The integrals on the right hand side can further be estimated by

$$
\begin{aligned}
\int_{l_{j}^{2} \leqq r^{2} \text { in } S} d u \frac{\left|M_{\alpha}\right|}{\prod_{S}\left(l_{j}^{2}\right)^{n_{j}}} & =r^{d} \int_{l_{j}^{2} \leqq 1 \text { in } S} d u \frac{\left|M_{\alpha}\right|}{\prod_{S}\left(l_{j}^{2}\right)^{n_{j}}} \\
& \leqq r^{d} \int_{u_{i}^{2} \leqq 1} d u \frac{\left|M_{\alpha}\right|}{\prod_{S}\left(l_{j}^{2}\right)^{n_{j}}}
\end{aligned}
$$

where $d$ is the dimension of the integral. Thus

$$
|J| \leqq \sum_{S \alpha} C_{S \alpha} \int_{u_{i}^{2} \leqq 1} d u \frac{\left|M_{\alpha}\right|}{\prod s\left(l_{j}^{2}\right)^{n_{j}}}
$$

with the sum restricted to those $M_{\alpha}$ which occur in (3.25) with non-vanishing coefficient. According to the Lemma (page 8 of this paper) we have convergence of the integrals on the right hand side if the dimension of any section is positive. In order to check the dimension $d$ of the full integral

$$
I=\int_{u_{i}^{2} \leqq 1} d u \frac{M_{\alpha}}{\prod_{s}\left(l_{j}^{2}\right)^{n_{j}}}
$$

we form the subintegral

$$
J(H)=\int d u_{1} \ldots d u_{a} \frac{P}{\prod\left(l_{j}^{2}+m_{j}^{2}\right)^{n_{j}}}
$$


of (3.4) along a hyperplane $H$ defined by constant values of $v_{1}, \ldots, v_{b}$. By the hypothesis $(3.2-3)$ the lower dimension $\delta$ of $J(H)$ is positive,

$$
\delta=4 a+\underline{\operatorname{deg}}_{u} P-\underline{\operatorname{deg}}_{u} \prod_{j}\left(l_{j}^{2}+m_{j}^{2}\right)^{n_{j}}>0 .
$$

This implies

$$
\begin{aligned}
0<\delta & =4 a+\underline{\operatorname{deg}}_{u} P-\operatorname{deg} \prod_{S}\left(l_{j}^{2}\right)^{n_{J}}-\operatorname{deg}_{u} \prod_{T}\left(l_{j}^{2}\right)^{n_{J}} \\
& \leqq 4 a+\underline{\operatorname{deg}_{u}} P-\operatorname{deg} \prod_{s}\left(l_{j}^{2}\right)^{n_{j}} \\
& \leqq 4 a+\operatorname{deg} M_{\alpha}-\operatorname{deg} \prod_{S}\left(l_{j}^{2}\right)^{n_{J}}=d .
\end{aligned}
$$

Hence the dimension of (3.32) is positive.

We further have to verify that the dimension $d_{i_{1} \ldots i_{c}}$ of each section

$$
I_{i_{1} \ldots i_{c}}=\int d u_{i_{1}} \ldots d u_{i_{c}} \frac{M_{\alpha i_{1} \ldots i_{c}}}{\prod_{i_{1} \ldots i_{c}}\left(l_{j}^{2}\right)^{n_{j}}}
$$

is positive. Here $M_{\alpha i_{1} \ldots i_{c}}$ is the restriction of the product (3.25) to factors depending on $u_{i_{1}} \ldots u_{i_{\alpha}} . \prod_{i_{1} \ldots i_{c}}$ denotes the product over all factors for which $l_{j} \in S$ is a linear combination of the vectors $u_{i_{1}} \ldots u_{i_{c}}$ only. Useful information is obtained by comparing the expansion

$$
P=\sum C_{\alpha} M_{\alpha}
$$

of $P$ with respect to monomials $M_{\alpha}$ in $u_{1}, \ldots, u_{a}$ with the expansion

$$
P=\sum C_{\alpha^{\prime}}^{\prime} M_{\alpha^{\prime}}^{\prime}
$$

with respect to independent monomials $M_{\alpha^{\prime}}^{\prime}$ in $u_{i_{1}} \ldots u_{i_{c}}$ only. We know that $M_{\alpha i_{1} \ldots i_{c}}$ occurs as a factor of at least one monomial $M_{\alpha}$ with $C_{\alpha} \neq 0$. Since the monomials $M_{\alpha}$ are linearly independent the factor $C_{\alpha^{\prime}}^{\prime}$ of $M_{\alpha^{\prime}}^{\prime}=M_{\alpha i_{1} \ldots i_{c}}$ in (3.36) must also be different from zero. This implies the inequality

$$
\underline{\operatorname{deg}}_{u^{\prime}} P \leqq \operatorname{deg} M_{\alpha i_{1} \ldots i_{c}}
$$

which will be crucial for the proof of the theorem. $\operatorname{deg}_{u^{\prime}}$ denotes the lower degree with respect to the variables $u^{\prime}=\left(u_{i_{1}}, \ldots, u_{i_{c}}\right)$. We now form the subintegral

$$
J\left(H^{\prime}\right)=\int d u_{i_{1}} \ldots d u_{i_{c}} \frac{P}{\prod_{j}\left(l_{j}^{2}+m_{j}^{2}\right)^{n_{j}}}
$$

along a hyperplance $H^{\prime}$ defined by constant values of $v_{1}, \ldots, v_{b}$ and the momenta $u_{j}$ which do not belong to $u^{\prime}$. The lower dimension $\delta^{\prime}$ of (3.38) is positive by hypothesis (3.2-3),

$$
\begin{aligned}
0<\delta^{\prime} & =4 c+\underline{\operatorname{deg}}_{u^{\prime}} P-\underline{\operatorname{deg}}_{u^{\prime}} \prod_{j}\left(l_{j}^{2}+m_{j}^{2}\right)^{n_{j}}, \\
& \leqq 4 c+\underline{\operatorname{deg}}_{u^{\prime}} P-\operatorname{deg} \prod_{i_{1} \ldots i_{c}}\left(l_{j}^{2}\right)^{n_{j}} .
\end{aligned}
$$

With (3.37)

$$
\begin{aligned}
d_{i_{1} \ldots i_{c}} & =4 c+\operatorname{deg} M_{\alpha i_{1} \ldots i_{c}}-\operatorname{deg} \prod_{i_{1} \ldots i c}\left(l_{j}^{2}\right)^{n_{j}} \\
& \geqq 4 c+\underline{\operatorname{deg}}_{u^{\prime}} P-\operatorname{deg} \prod_{i_{1} \ldots i_{c}}\left(l_{j}^{2}\right)^{n_{j}}
\end{aligned}
$$


follows. Hence the dimension $d_{i_{1} \ldots i_{c}}$ of (3.38) is positive. According to the lemma each integral on the right hand side of (3.30) converges. This completes the proof of the theorem.

\section{Reduced Integrals}

In this section we discuss integrals of the form

$$
I=\int d k \prod_{j=1}^{n} \Delta_{j}\left(l_{j}\right)
$$

in the notation of (1.1) and

$$
\Delta_{j}\left(l_{j}\right)=\frac{M_{j}}{\left(l_{j}^{2}-m_{j}^{2}+i \varepsilon\left(\vec{l}_{j}^{2}+m_{j}^{2}\right)\right)^{n_{j}}}, \quad n_{j}>0,
$$

where $M_{j}$ is a monomial in $k$ and $p$. For integrals of this type we introduce the concept of the reduced integral. Let

$$
S=\left(l_{j_{1}}, \ldots, l_{j_{c}}\right)
$$

be any subset of the momenta $l_{1}, \ldots, l_{n}$. From the elements of $S$ we select a basis, i.e. we choose linearly independent forms $u_{1}, \ldots, u_{a}$ of $L$ such that each $l_{j} \in S$ is a linear combination of $u_{1}, \ldots, u_{a}$ and $p_{1}, \ldots, p_{N}$. With respect to $S$ we form the reduced integral

$$
\begin{aligned}
& I_{\text {red }}(S) \propto \int d u_{1} \ldots d u_{a} \prod_{s} \Delta_{j}\left(l_{j}\right) \\
& l_{j}=l_{j}(u, p), \quad u=\left(u_{1}, \ldots, u_{a}\right)
\end{aligned}
$$

where the product $\prod_{S}$ extends over the $l_{j} \in S$ only. The reduced integral (4.4) is defined up to a factor which depends on the chosen basis.

Of special interest are reduced integrals of vanishing masses and vanishing external momenta, i.e.

$$
\begin{aligned}
m_{j} & =0 \quad \text { if } \quad l_{j} \in S, \\
l_{j} & =l_{j}(u), \quad \text { independent of } p, \text { if } l_{j} \in S .
\end{aligned}
$$

In this case each factor $\Delta_{j}\left(l_{j}\right)$ occurring in the reduced integral is homogeneous in $u$.

In case that (4.1) represents an unrenormalized Feynman integral the reduced integrals have a simple graphical interpretation: $I_{\text {red }}(S)$ is the Feynman integral which corresponds to the reduced diagram $S^{\prime}=S / T$ where all lines of $T$ have been contracted to a point.

With the concept of the reduced integral we can give an equivalent formulation of the infrared convergence condition for integrals of type $(4.1)^{1}$. Consider a basis

$$
u_{1}=l_{i_{1}}, \ldots, u_{a}=l_{i_{a}}, \quad v_{1}=l_{j_{1}}, \ldots, v_{b}=l_{j_{b}}
$$

of $L$ with

$$
m_{i_{1}}=\ldots=m_{i_{a}}=0 .
$$

1 This formulation was used by Mack [14] to study infrared convergence of integrals like (4.1) in the context of conformally in variant theorems. 
For any such basis the infrared convergence condition reads

$$
\underline{\operatorname{deg}}_{u} \prod \Delta_{j}\left(l_{j}\right)+4 a>0 .
$$

We now form the reduced integral

$$
I_{\text {red }}(S) \propto \int d u \prod_{s} \Delta_{j}\left(l_{j}\right)
$$

with respect to the set $S$ of all momenta $l_{j}$ with $m_{j}=0$ and $l_{j}=0$ at $u=0$. Then for any $l_{j} \notin S$ we have

$$
\left.\begin{array}{rl}
m_{j} \neq 0 & \\
\text { or } \quad l_{j} \neq 0 \quad \text { at } \quad u=0
\end{array}\right\} \text { if } l_{j} \notin S .
$$

Therefore,

$$
\operatorname{deg}_{u} \prod \Delta_{j}\left(l_{j}\right)=\operatorname{deg} \prod_{s} \Delta_{j}\left(l_{j}\right)
$$

and

$$
\underline{\operatorname{deg}_{u}} \prod \Delta_{j}\left(l_{j}\right)+4 a=\operatorname{dim} I_{\text {red }}(S) .
$$

Hence an equivalent formulation of the infrared convergence condition for the integral (4.1) is

$$
\operatorname{dim} I_{\text {red }}(S)>0
$$

for any set $S$ of momenta $l_{j}$ with

$$
\left.\begin{array}{rl}
m_{j} & =0 \\
\text { and } \quad l_{j}=0 \quad \text { at } \quad u=0
\end{array}\right\} \text { if } l_{j} \in S .
$$

With this result, we are able to formulate the infrared convergence condition for an integral of the type [notation of (1.1) and (4.1)]

$$
\int d k Q \prod_{j=1}^{n} \Delta_{j}\left(l_{j}\right)
$$

where $Q$ is a polynomial in $k$ and $p$, in terms of a power counting criterion involving the formal integral

$$
\int d k \prod_{s_{0}} \Delta_{j}\left(l_{j}\right)
$$

where the product is restricted to the set $S_{0}$ of momenta with $m_{j}=0$. In particular, we have the following

Corollary to the Power Counting Theorem. The integral (4.13) is absolutely convergent if the ultraviolet convergence condition (3.1) holds and if any reduced integral of (4.14) with vanishing external momenta has positive dimension.

Proof.

$$
\begin{aligned}
\underline{\operatorname{deg}}_{u} Q \prod_{j=1}^{n} \Delta_{j}\left(l_{j}\right) & =\underline{\operatorname{deg}_{u}} Q+\underline{\operatorname{deg}_{u}} \prod_{j=1}^{n} \Delta_{j}\left(l_{j}\right) \\
& \geqq \underline{\operatorname{deg}}_{u} \prod_{j=1}^{n} \Delta_{j}\left(l_{j}\right) .
\end{aligned}
$$


Hence the infrared convergence condition of (4.13) is implied by that of (4.1). Any reduced integral of (4.1) with (4.12) is also a reduced integral of (4.14). This completes the proof.

\title{
References
}

1. Dyson, F. J.: Phys. Rev. 75, 1736 (1949)

2. Weinberg, S. : Phys. Rev. 118, 838 (1960)

3. Bogoliubov, N.N., Shirkov,D.V.: Introduction to the theory of quantized fields. New York: Interscience 1959

4. Gomes, M., Lowenstein, J., Zimmermann, W.: Commun. math. Phys. 39, 81 (1974)

5. Lowenstein, J.: in preparation

6. Lowenstein, J., Zimmermann, W. : to be published

7. Clark, T.: to be published

8. Becchi, C.: in preparation

9. Lowenstein, J.: in preparation

10. Bergère, M., Lam, P.: Commun. math. Phys. 39, 1 (1974) and to be published

11. Trate, H.: DESY preprint $74 / 44$ (1974)

12. Pohlmeyer, K.: DESY preprint 74/36 (1974)

13. Hahn, Y., Zimmermann, W.: Commun. math. Phys. 10, 330 (1968)

14. Mack, G.: In scale and conformal symmetry in hadron physics, ed. R. Gatto, New York: J. Wiley 1973

Communicated by K. Symanzik

\author{
J. H. Lowenstein \\ Physics Department \\ New York University \\ New York, N. Y., USA \\ W. Zimmermann \\ Max-Planck-Institut \\ für Physik und Astrophysik \\ D-8000 München 4 \\ Föhringer Ring 6 \\ Federal Republic of Germany
}

